\title{
Herbal Medicine Formulas for Parkinson's Disease: A Systematic Review and Meta-Analysis of Randomized Double-Blind Placebo-Controlled Clinical Trials
}

\section{OPEN ACCESS}

Edited by:

Seung-Nam Kim,

Dongguk University Seoul,

South Korea

Reviewed by:

Hyangsook Lee,

Kyung Hee University, South Korea

Seung-Yeon Cho,

Kyung Hee University, South Korea

Song-Yi Kim,

Gachon University, South Korea

*Correspondence:

Yan Lin

linyanm@vip.sina.com

Guo-Qing Zheng

gq_zheng@sohu.com

tThese authors have contributed equally to this work

Received: 07 June 2018 Accepted: 15 October 2018 Published: 08 November 2018

Citation:

Shan C-S, Zhang H-F, Xu Q-Q,

Shi Y-H, Wang Y, Li Y, Lin Y and Zheng G-Q (2018) Herbal Medicine Formulas for Parkinson's Disease: A Systematic Review and Meta-Analysis of Randomized Double-Blind

Placebo-Controlled Clinical Trials. Front. Aging Neurosci. 10:349. doi: 10.3389/fnagi.2018.00349

\section{Chun-Shuo Shan ${ }^{\dagger}$, Hong-Feng Zhang ${ }^{\dagger}$, Qing-Qing Xu, Yi-Hua Shi, Yong Wang, Yan Li, Yan Lin* and Guo-Qing Zheng * \\ Department of Neurology, The Second Affiliated Hospital and Yuying Children's Hospital of Wenzhou Medical University,
Wenzhou, China}

Background: Parkinson's disease (PD) is a debitlitating, chronic, progressive neurodegenerative disorder without modifying therapy. Here, we aimed to evaluate the available evidence of herbal medicine (HM) formulas for patients with PD according to randomized double-blind placebo-controlled clinical trials.

Methods: HM formulas for PD were searched in eight main databases from their inception to February 2018. The methodological quality was assessed using Cochrane Collaboration risk of bias tool. Meta-analysis was performed using RevMan 5.3 software.

Results: Fourteen trials with Seventeen comparisons comprising 1,311 patients were identified. Compared with placebo groups, HM paratherapy ( $n=16$ comparisons) showed significant better effects in the assessments of total Unified Parkinson's Disease Rating Scale (UPDRS) (WMD: $-5.43,95 \% \mathrm{Cl}-8.01$ to $-2.86 ; P<0.0001)$, UPDRS I (WMD: $-0.30,95 \% \mathrm{Cl}:-0.54$ to $-0.06 ; P=0.02)$, UPDRS || (WMD: -2.21 , 95\% Cl: -3.19 to $-1.22 ; P<0.0001)$, UPDRS III (WMD: $-3.26,95 \% \mathrm{Cl}:-4.36$ to -2.16; $P<0.00001)$, Parkinson's Disease Quality of Life Questionnaire $(p<0.01)$ and Parkinson's Disease Questionnaire-39 (WMD: $-7.65,95 \% \mathrm{Cl}$ : -11.46 to -3.83 ; $p<0.0001)$, Non-motor Symptoms Questionnaire $(p<0.01)$ and Non-Motor Symptoms Scale (WMD: -9.19 , 95\% Cl: -13.11 to $-5.28 ; P<0.00001)$, Parkinson's Disease Sleep Scale (WMD: 10.69, 95\% Cl: 8.86 to 12.53; $P<0.00001$ ), and Hamilton depression rating scale (WMD: $-5.87,95 \% \mathrm{Cl}:-7.06$ to $-4.68 ; P<0.00001)$. The efficiency of HM monotherapy ( $n=1$ comparison) was not superior to the placebo according to UPDRS II, UPDRS III and total UPDRS score in PD patients who never received levodopa treatment, all $P>0.05$. HM formulas paratherapy were generally safe and well tolerated for PD patients (RR: $0.41,95 \% \mathrm{Cl}$ : 0.21 to $0.80 ; P=0.009$ ).

Conclusion: The findings of present study supported the complementary use of HM paratherapy for PD patients, whereas the question on the efficacy of HM monotherapy in alleviating PD symptoms is still open.

Keywords: Parkinson's disease, randomized double-blind placebo-controlled clinical trial, traditional Chinese medicine, meta-analysis, systematic review 


\section{INTRODUCTION}

Parkinson's disease (PD) is a common chronic neurodegenerative disease characterized by the degeneration of dopaminergic neurons in the substantia nigra (SN) (Kalia and Lang, 2015), and presents with non-motor or/and motor syndrome (Rogers et al., 2017). In the Global Burden of Diseases, Injuries, and Risk Factors Study (GBD) 2016, PD was the second leading cause in neurological disorders of years lived with disability (YLDs), contributing to 6.1 million of patients (GBD, 2016 Disease and Injury Incidence and Prevalence Collaborators, 2017). From 2005 to 2015 , global deaths due to PD increased by $42.4 \%$, to 117.4 thousands deaths (GBD, 2015 Mortality and Causes of Death Collaborators, 2016), as a result of population aging. With the growing incidence, PD seriously hurt the physical and mental health of the elderly, also produced a heavy economic burden on both families and society. The average annual cost per PD patient was $\$ 22,800$ in the United States (Kowal et al., 2013) and \$36,085 in the UK (Findley et al., 2011). Current conventional treatment for $\mathrm{PD}$ is based on the dopamine (DA) replacement therapies and reduction of DA degradation, including levodopa, DA receptor agonists, monoamine oxidase-B inhibitors, catechol-Omethyltransferase inhibitors and other types of drugs (Rogers et al., 2017). However, all the current therapeutic approaches remain palliative and can't inhibit or reverse the progression of PD (Athauda and Foltynie, 2015). Furthermore, frequently with these treatments can lead to obvious adverse events and efficacies diminished, as well as induce therapy-related motor complications such as dyskinesia, choreoathetosis, and fluctuations in motor function (Jenner, 2015). A safer and more effective alternative treatment of PD is increasingly demanded.

The therapy of herbal medicine (HM) for PD is particularly common. In China, HM could be traced in the Huangdi Neijing (Inner Canon of Yellow Emperor) (Zheng, 2009), the earliest existing classics in Chinese herbal medicine (CHM). Up to now, $\mathrm{HM}$ is still very popular in the treatment of PD especially in Asian countries (Wang et al., 2011, 2013). Previous reviews (Wang et al., 2012; Zhang et al., 2015) found lack of evidence of supporting the use of HM for PD patients because of the generally low-quality studies included. Here, we performed a systematic review and meta-analysis of randomized double-blind placebocontrolled clinical trials of HM formulas for PD patients and further explored the mechanisms of high-frequently used herbs against PD.

\section{METHODS}

This systematic review and meta-analysis is conducted according to the Preferred Reporting Items for Systematic Reviews and Meta-Analyses: The PRISMA Statement (Moher et al., 2010b) and our previous study (Yang et al., 2017).

\section{Search Strategy}

Randomized double-blind placebo-controlled clinical trials of $\mathrm{HM}$ formulas for PD were searched in eight databases from their inception to February 2018. They are PubMed, EMBASE, Cochrane Central Register of Controlled Trials
(CENTRAL), Web of science, Chinese National Knowledge Infrastructure (CNKI), Chinese VIP Information, Wanfang database and Chinese Biological Medical Literature Database (CBM). Moreover, we hand searched additional relevant studies using the reference list of previous reviews. The search strategy of PubMed was as follows, and was modified to suit other English or Chinese databases.

PubMed search strategy:

\#1. Parkinson disease $[\mathrm{mh}]$

\#2. Parkinson*[tiab]

\#3. \#1OR \#2

\#4. Medicine, Chinese Traditional [mh]

\#5. Herbal Medicine [mh]

\#6. Integrative Medicine [mh]

\#7. traditional Chinese medicine [tiab]

\#8. herb* [tiab]

\#9. \#4 OR \#5 OR \#6 OR \#7 OR \#8

\#10. \#3 And \#9

\section{Study Selection}

Two authors (CS-S and H-FZ) independently engaged in the selection of studies by reading study titles, abstracts and full texts. The disagreement was resolved by the corresponding author (GZ) or repeated discussion.

\section{Inclusion Criteria}

Type of study: the articles were randomized double-blind placebo-controlled clinical trials.

Type of participants: participants were of any age or sex with a confirmed diagnosis of PD according to the UK Brain Bank criteria (Hughes et al., 1992), Chinese National Diagnosis Standard (CNDS) for PD in 1984 (Wang, 1985), CNDS updated version in 2006 for PD (Zhang, 2006) or other formal comparable criteria.

Type of intervention: Analyzed interventions were HM formulas or HM formulas plus western conventional medicine (WCM) according to $\mathrm{PD}$ treatment guidelines, ${ }^{2}$ regardless of the form of the drug, dosage, frequency or duration of the treatment. Comparator interventions were placebo or placebo plus WCM.

Type of outcome measures: the primary outcomes were total Unified Parkinson's Disease Rating Scale (UPDRS) score, UPDRS I (Mental Score), UPDRS II (Activities of Daily Life), UPDRS III (Motor Score), and UPDRS IV (Complications of treatment). The secondary outcomes were: (1) Parkinson's Disease Quality of Life Questionnaire (PDQL) and Parkinson's Disease Questionnaire-39 (PDQ-39); (2) Non-motor Symptoms Questionnaire (NMSQuest) and NonMotor Symptoms Scale (NMSS); (3) Parkinson's Disease Sleep Scale (PDSS); (4) Hamilton depression rating scale (HAMD); (5) Adverse events.

\section{Exclusion Criteria}

Studies were excluded if they were any one of the followings: (1) clinical trials evaluating the other alternative and complementary medicines mixed in the experimental group or control group 
in the treatment of PD; (2) single herb, herbal extracts and herbal components; (3) case series, reviews, comments and protocols; (4) animal studies and in vitro studies; (5) duplicated publications.

\section{Quality Assessment}

The methodological quality was evaluated by using the Cochrane Collaboration's risk of bias tool (Higgins et al., 2011). The quality of each study was assessed by following seven biases: adequate sequence generation, allocation concealment, blinding of participants and personnel, blinding of outcome assessors, incomplete outcome data addressed (ITT analysis), free of selective reporting and other bias. Each domain can be rated as “+” (low risk of bias), “-” (high risk of bias), or "?” (unclear risk of bias), which were the three categories for the degree of each potential bias.

\section{Data Extraction}

Two authors (CS-S and HF-Z) independently extracted the data according to predefined extraction form as follows: (1) General information: the first author's name, publication year, and publication language; (2) Participants: diagnostic criteria, study design, total number and number in comparison groups, gender and mean age; (3) Intervention: herbal preparations, dose, frequency, course of treatment, followup; (4) Outcome measures. If the study had multiple comparison groups, we chosen the most relevant groups for analysis. The original authors were contacted if further information was needed. Disagreements were resolved through discussing with corresponding author (GZ).

The constituent of HM formulas for PD in each included study was recorded. The herbs with cumulative frequencies over $50 \%$ are documented and ranked.

\section{Data Synthesis and Statistical Analysis}

We synthesized all data and performed meta-analyses on RevMan 5.3 software. Continuous outcomes were using weighted mean differences (WMD) or standardized mean differences (SMD) with 95\% confidence intervals (CIs), while dichotomous outcomes were summarized using risk ratio (RR) with 95\% confidence intervals (CIs). Heterogeneity among studies was detected by $\mathrm{I}^{2}$ and $\mathrm{Chi}^{2}$ tests. If substantial statistical heterogeneity existed ( $\left.I^{2} \geq 50 \%, P<0.10\right)$, a random-effects model was used. If there was no observed heterogeneity $\left(I^{2}\right.$ $<50 \%, P>0.10$ ), a fixed-effect model was applied. Possible sources of heterogeneity were explored by subsequent sensitivity analyses. If more than ten trials were identified in each outcome, publication bias was detected by funnel plot analyses and Egger's test.

\section{RESULTS}

\section{Description of the Screening Process}

The detailed screening process was summarized in the PRISMA flow diagram (Figure 1). A total of 7,521 potentially relevant hits were initially yielded from the eight databases and other sources, in which 6,570 records were remained after removal of duplicates.
Through screening titles and abstracts, we excluded 5,824 papers because they were studies with no relevance to PD $(n=3292)$, nonclinical trials $(n=1007)$, case reports, reviews, comments OR protocols $(n=1525)$. After full-text evaluation, 732 papers were excluded, including 234 that were not CHM studies, 142 that contained mixed interventions, 38 that aimed at single herb, herbal extracts or components, 305 that were not randomized double-blind placebo-controlled trials, and 13 that observed no outcome of interest. Ultimately, 14 eligible studies (Pan et al., 2009, 2011, 2013; Zhao et al., 2009, 2013; Guo, 2010; Kum et al., 2011; Chen M. Y. et al., 2014; Guo et al., 2014; Wen et al., 2015; Li et al., 2016; Yu, 2016; Cai et al., 2017; Yang, 2017) were selected in our study.

\section{STUDY CHARACTERISTICS}

The general characteristics of the included studies are summarized in Table 1. Fourteen included studies (Pan et al., 2009, 2011, 2013; Zhao et al., 2009, 2013; Guo, 2010; Kum et al., 2011; Chen M. Y. et al., 2014; Guo et al., 2014; Wen et al., 2015; Li et al., 2016; Yu, 2016; Cai et al., 2017; Yang, 2017) were published between 2009 and 2017. Among them, 4 studies (Kum et al., 2011; Pan et al., 2011, 2013; Li et al., 2016) were published in English and 10 studies (Pan et al., 2009; Zhao et al., 2009, 2013; Guo, 2010; Chen M. Y. et al., 2014; Guo et al., 2014; Wen et al., 2015; Yu, 2016; Cai et al., 2017; Yang, 2017) in Chinese. The most used diagnostic criterion of PD was UK Brain Bank criteria, which was referred in 11 studies (Guo, 2010; Kum et al., 2011; Pan et al., 2011, 2013; Zhao et al., 2013; Chen M. Y. et al., 2014; Guo et al., 2014; Wen et al., 2015; Li et al., 2016; Cai et al., 2017; Yang, 2017). Comparison of HM monotherapy vs. placebo was performed in one trial (Zhao et al., 2009). Comparisons of CHM plus WCM versus placebo plus WCM were conducted in 14 trials (Pan et al., 2009, 2011, 2013; Zhao et al., 2009, 2013; Guo, 2010; Kum et al., 2011; Chen M. Y. et al., 2014; Guo et al., 2014; Wen et al., 2015; Li et al., 2016; Yu, 2016; Cai et al., 2017; Yang, 2017), of whom 7 trials used Madopar (Zhao et al., 2009; Guo, 2010; Guo et al., 2014; Li et al., 2016; Yu, 2016; Cai et al., 2017; Yang, 2017). All studies involved a total of 1,311 patients with 675 in the treatment group vs. 636 in the placebo group, ranging in age from 51 to 79 years old. The sample size of the included studies ranged from 47 to 242 . The male-to-female ratio was between 1.0 and 2.1. Duration of disease ranged from 2.2 months to 11.3 years. The total intervention period varied from 8 weeks to 6 months. The most common duration was 12 weeks. Two studies (Pan et al., 2013; Li et al., 2016) mentioned the follow-up times were 4 weeks and 6 months, respectively.

\section{Description of the HM Formulas}

Fourteen studies reported a wide range of TCM formulas, including Bushen Huoxue granule $(n=2)$, Zengxiao Anshen Zhichan 2 capsule $(n=1)$, Zengxiao Anshen Zhichan 2 granule $(n=1)$, Zhichan decoction $(n=1)$, Zhichan granule $(n=1)$, Guilu Dihuang capsule $(n=1)$, Jiawei Liujunzi Tang granule $(n=1)$, Yangxue Qingnao granule $(n=1)$, Congwu Qufeng granule $(n=1)$, Yishen Chuchan decoction $(n=1)$, Naokang granule $(n=1)$, Guiling Pa'an capsule $(n=1)$, and Guiling 


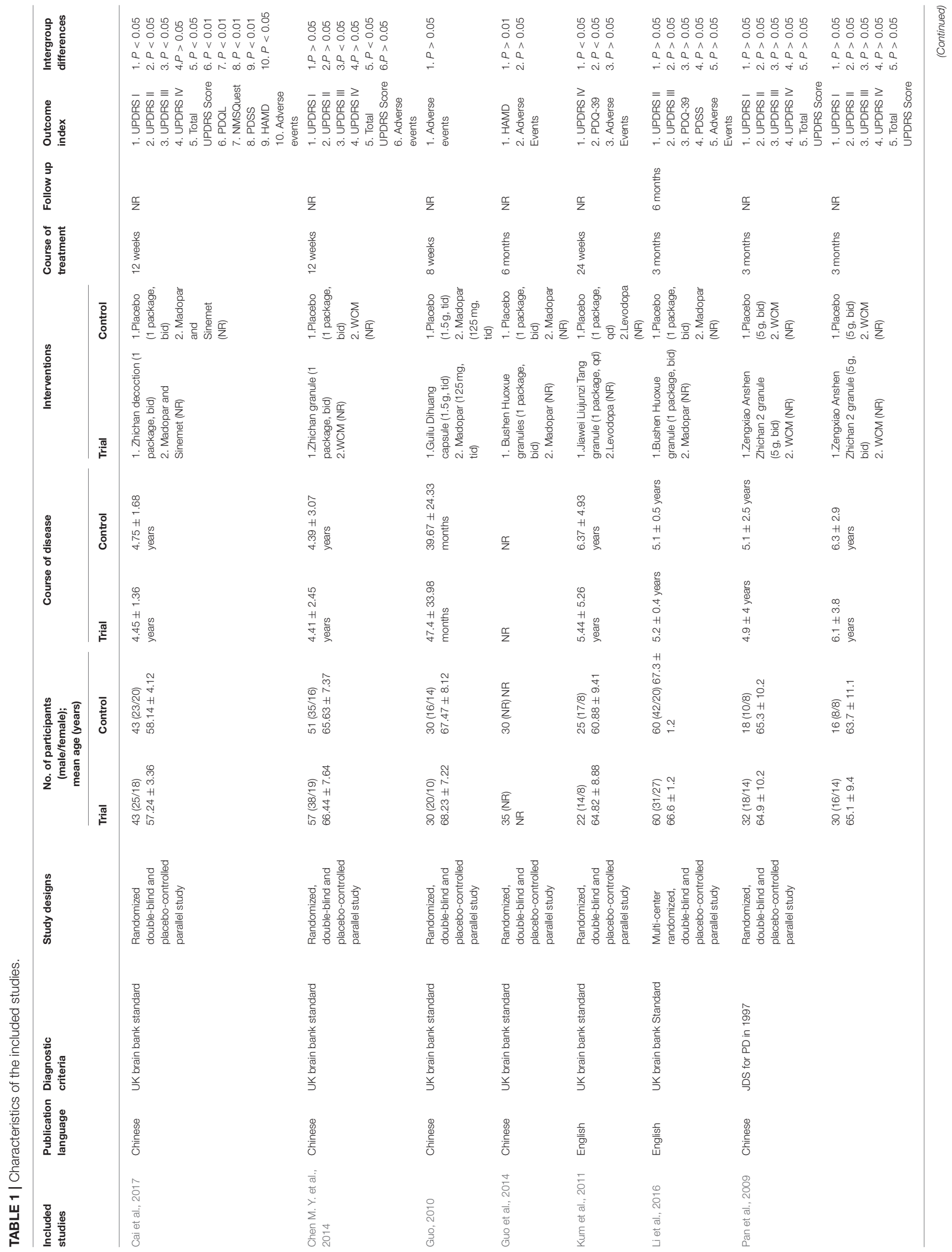




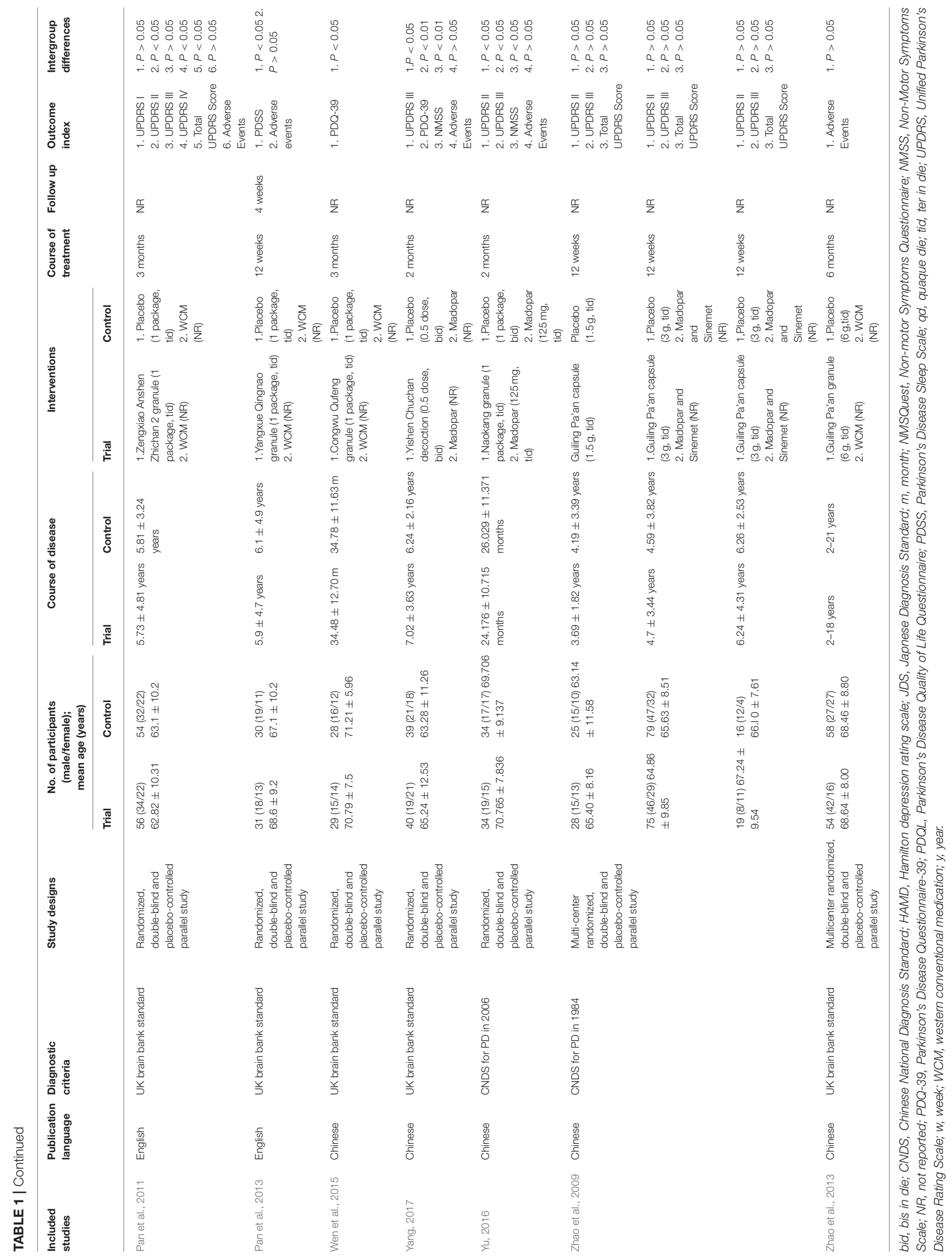




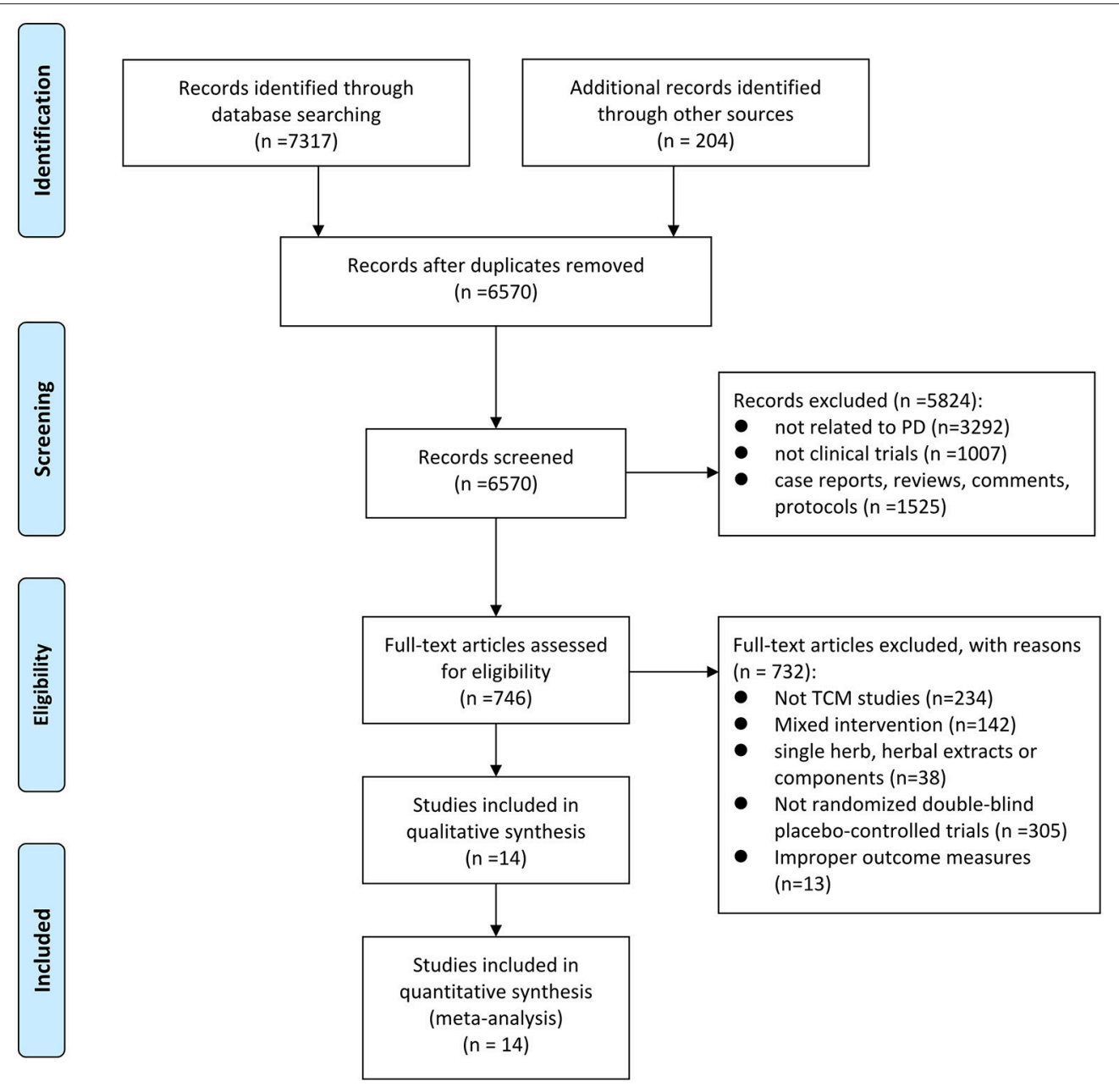

FIGURE 1 | Flow diagram of the search process.

Pa'an granule $(n=1)$. The ingredients of TCM formulas in each included studies were presented in Table 2. A total of 52 herbs were used in these TCM formulas. High-frequency herbs in HM formulas were ranked in Table 3. The top 11 most frequently used herbs were Radix Salviae Miltiorrhizae (Dan Shen), Radix Paeoniae Alba (Bai Shao), Ramulus Uncariae Cum Uncis (Gou Teng), Radix Rehmanniae (Di Huang), Herba Cistanches (Rou Cong Rong), Radix Polygoni Multiflori (He Shou Wu), Rhizoma Ligustici Chuanxiong (Chuan Xiong), Fructus Corni (Shan Zhu Yu), Radix Angelicae Sinensis (Dang Gui), Rhizoma Acori Tatarinowii (Shi Chang Pu), and Radix Astragali seu Hedysari (Huang Qi).

\section{Assessing the Quality of Studies}

The methodological quality of all included studies was detailed in the Figure 2. All included studies were randomized studies with explicit description. Specifically, seven studies (Pan et al., 2009, 2011; Guo et al., 2014; Wen et al., 2015; Yu, 2016; Cai et al., 2017; Yang, 2017) used random number tables. Four studies (Zhao et al., 2009; Kum et al., 2011; Pan et al., 2013; Chen M. Y. et al., 2014) used computer-generated lists of random numbers. Two studies (Zhao et al., 2013; Li et al., 2016) employed online center distribution, while only one study (Guo, 2010) stated the method for sequence generation by simple randomization. Eleven studies (Zhao et al., 2009, 2013; Kum et al., 2011; Pan et al., 2011, 2013; Chen M. Y. et al., 2014; Wen et al., 2015; Li et al., 2016; Yu, 2016; Cai et al., 2017; Yang, 2017) reported adequate allocation concealment. Four (Kum et al., 2011; Chen M. Y. et al., 2014; Yu, 2016; Cai et al., 2017) adopted opaque and sealed envelopes. The remaining studies (Zhao et al., 2009, 2013; Pan et al., 2011, 2013; Wen et al., 2015; Li et al., 2016; Yang, 2017) adopted center distribution. Of 14 included studies, 8 studies (Pan et al., 2009; Zhao et al., 2009, 2013; Guo, 2010; Guo et al., 2014; Wen et al., 2015; Yu, 2016; Yang, 2017) applied double blinding and 6 studies (Kum et al., 2011; Pan et al., 2011, 2013; Chen M. Y. et al., 2014; Li et al., 2016; Cai et al., 2017) had triple blinding. All studies had low risk of bias in the incomplete out-come data. Four studies (Guo, 2010; Guo et al., 2014; Yu, 2016; Yang, 2017) had unclear risk of bias in selective reporting because of no available protocols. Other 
TABLE 2 | Ingredients of TCM formula.

\begin{tabular}{|c|c|c|c|c|}
\hline \multirow[t]{2}{*}{ Included studies } & \multirow[t]{2}{*}{ Prescription } & \multicolumn{3}{|c|}{ Ingredients } \\
\hline & & Latin name & English name & Chinese name \\
\hline Cai et al., 2017 & Zhichan decoction & $\begin{array}{l}\text { Radix Astragali seu Hedysari, Radix } \\
\text { Paeoniae Alba, Radix Salviae } \\
\text { Miltiorrhizae, Rhizoma Anemarrhenae, } \\
\text { Ramulus Uncariae Cum Uncis, } \\
\text { Rhizoma Cimicifugae, Rhizoma } \\
\text { Polygoni Cuspidati. }\end{array}$ & $\begin{array}{l}\text { Milkvetch root, debark peony root, } \\
\text { danshen root, common anemarrhena } \\
\text { rhizome, gambir plant nod, } \\
\text { largetrifoliolious bugbane rhizome, } \\
\text { giant knotweed rhizome. }\end{array}$ & $\begin{array}{l}\text { Huang Qi, Bai Shao, Dan Shen, Zhi } \\
\text { Mu, Gou Teng, Sheng Ma, Hu Zhang. }\end{array}$ \\
\hline $\begin{array}{l}\text { Chen M. Y. et al., } \\
2014\end{array}$ & Zhichan granule & $\begin{array}{l}\text { Radix Astragali seu Hedysari, } \\
\text { Ramulus Uncariae Cum Uncis, Radix } \\
\text { Polygoni Multiflori Preparata, Radix } \\
\text { Paeoniae Alba, Rhizoma } \\
\text { Anemarrhenae, et al. }\end{array}$ & $\begin{array}{l}\text { Milkvetch root, gambir plant nod, } \\
\text { prepared fleeceflower root, debark } \\
\text { peony root, common anemarrhena } \\
\text { rhizome, et al. }\end{array}$ & $\begin{array}{l}\text { Huang Qi, Gou Teng, Zhi He Shou } \\
\text { Wu, Bai Shao, Zhi Mu, et al. }\end{array}$ \\
\hline Guo, 2010 & Guilu Dihuang capsule & $\begin{array}{l}\text { Radix Rehmanniae Preparata, } \\
\text { Chinemys reevesii, Colla Corni Cervi, } \\
\text { et al. }\end{array}$ & $\begin{array}{l}\text { Prepared rehmannia root, tortoise } \\
\text { plastron glue, deerhorn glue, et al. }\end{array}$ & $\begin{array}{l}\text { Shu Di Huang, Gui Ban Jiao, Lu Jiao } \\
\text { Jiao, et al. }\end{array}$ \\
\hline Guo et al., 2014 & $\begin{array}{l}\text { Bushen Huoxue } \\
\text { granule }\end{array}$ & $\begin{array}{l}\text { Fructus Corni, Herba Cistanches, } \\
\text { Radix Polygoni Multiflori, Rhizoma } \\
\text { Ligustici Chuanxiong, Radix } \\
\text { Angelicae Sinensis, Radix Salviae } \\
\text { Miltiorrhizae, Scolopendra, et al. }\end{array}$ & $\begin{array}{l}\text { Asiatic cornelian cherry fruit, } \\
\text { desertliving cistanche, fleeceflower } \\
\text { root, sichuan lovage rhizome, } \\
\text { Chinese angelica, danshen root, } \\
\text { centipede, et al. }\end{array}$ & $\begin{array}{l}\text { Shan Zhu Yu; Rou Cong Rong; He } \\
\text { Shou Wu; Chuan Xiong; Dang Gui; } \\
\text { Dan Shen; Wu Gong , et al. }\end{array}$ \\
\hline Kum et al., 2011 & $\begin{array}{l}\text { Jiawei Liujun Zi Tang } \\
\text { granule }\end{array}$ & $\begin{array}{l}\text { Radix Codonopsis, Radix } \\
\text { Rehmanniae Recens, Poria, Ramulus } \\
\text { Uncariae Cum Uncis, Rhizoma } \\
\text { Atractylodis Macrocephalae, Radix } \\
\text { Angelicae Sinensis, Rhizoma Pinelliae } \\
\text { Preparatum, Rhizoma Ligustici } \\
\text { Chuanxiong, Radix Achyranthis } \\
\text { Bidentatae, Pericarpium Citri } \\
\text { Reticulatae, Radix Glycyrrhizae. }\end{array}$ & $\begin{array}{l}\text { Tangshen, unprocessed rehmannia } \\
\text { root, Indian bread, gambir plant nod, } \\
\text { largehead atractylodes rhizome, } \\
\text { Chinese angelica, processed pinellia } \\
\text { tuber, sichuan lovage rhizome, } \\
\text { twotoothed achyranthes root, dried } \\
\text { tangerine peel, liquorice root. }\end{array}$ & $\begin{array}{l}\text { Dang shen; Sheng di huang; Fu ling; } \\
\text { Gou Teng; Bai Zhu; Dang Gui; Fa ban } \\
\text { xia; Chuan Xiong; niu xi; Chen pi; Gan } \\
\text { cao. }\end{array}$ \\
\hline Li et al., 2016 & $\begin{array}{l}\text { Bushen Huoxue } \\
\text { granule }\end{array}$ & $\begin{array}{l}\text { Fructus Corni, Rhizoma Acor } \\
\text { tatarinowii, Radix Polygoni multiflori, } \\
\text { Herba Cistanches, Raix Angelicae } \\
\text { sinensis, Radix Salviae miltiorrhizae, } \\
\text { Scolopendra. }\end{array}$ & $\begin{array}{l}\text { Asiatic cornelian cherry fruit, grassleaf } \\
\text { sweetflag rhizome, fleeceflower root, } \\
\text { desertliving cistanche, Chinese } \\
\text { angelica, danshen root, centipede. }\end{array}$ & $\begin{array}{l}\text { Shan Zhu Yu, Shi Chang Pu, He Shou } \\
\text { Wu; Rou Cong Rong, Dang Gui; Dan } \\
\text { Shen; Wu Gong. }\end{array}$ \\
\hline Pan et al., 2009 & $\begin{array}{l}\text { Zeng-xiao An-shen } \\
\text { Zhi-chan } 2 \text { capsule }\end{array}$ & $\begin{array}{l}\text { Radix Rehmanniae Preparata, } \\
\text { Fructus Corni, Os Draconis, Radix } \\
\text { Asparagi, Radix Paeoniae Alba, } \\
\text { Carapax et Plastrum Testudinis, } \\
\text { Herba Cistanches, Radix Puerariae, } \\
\text { Rhizoma Arisaematis Cum Bile, } \\
\text { Scorpio, Radix Salviae Miltiorrhizae, } \\
\text { Lumbricus, Rhizoma Acori Tatarinowii, } \\
\text { Rhizoma Curcumae Longae. }\end{array}$ & $\begin{array}{l}\text { Prepared rehmannia root, asiatic } \\
\text { cornelian cherry fruit, bone fossil of } \\
\text { big mammals, cochinchinese } \\
\text { asparagus root, debark peony root, } \\
\text { tortoise carapace and plastron, } \\
\text { desertliving cistanche, kudzuvine } \\
\text { root, bile arisaema, scorpion, } \\
\text { Danshen root, earthworm, grassleaf } \\
\text { sweetflag rhizome, turmeric. }\end{array}$ & $\begin{array}{l}\text { Shu Di Huang; Shan Zhu Yu; Long } \\
\text { Gu; Tian Dong; Shao Yao; Gui Jia; } \\
\text { Rou Cong Rong; Ge Gen; Dan Nan } \\
\text { Xing; Quan Xie; Dan Shen; Di Long; } \\
\text { Shi Chang Pu; Jiang Huang. }\end{array}$ \\
\hline Pan et al., 2011 & $\begin{array}{l}\text { Zengxiao Anshen } \\
\text { Zhichan } 2 \text { granule }\end{array}$ & $\begin{array}{l}\text { Ramulus Uncariae Cum Uncis, Radix } \\
\text { Rehmanniae Recens, Fructus Corni, } \\
\text { Radix Asparagi, Radix Paeoniae Alba, } \\
\text { Herba Cistanches, Radix Puerariae, } \\
\text { Rhizoma Arisaematis, Radix Salviae } \\
\text { Miltiorrhizae, Rhizoma Acori } \\
\text { Tatarinowii, Rhizoma Curcumae } \\
\text { Longae, Radix Morindae Officinalis, } \\
\text { Rhizoma Gastrodiae, Rhizoma } \\
\text { Ligustici Chuanxiong, }\end{array}$ & $\begin{array}{l}\text { Gambir plant nod, unprocessed } \\
\text { rehmannia root, asiatic cornelian } \\
\text { cherry fruit, cochinchinese asparagus } \\
\text { root, debark peony root, desertliving } \\
\text { cistanche, kudzuvine root, } \\
\text { jackinthepulpit tuber, danshen root, } \\
\text { grassleaf sweetflag rhizome, turmeric, } \\
\text { morinda root, tall gastrodia tuber, } \\
\text { sichuan lovage rhizome. }\end{array}$ & $\begin{array}{l}\text { Gou Teng; Sheng Di Huang; Shan } \\
\text { Zhu Yu; Tian Dong; Bai Shao; Rou } \\
\text { Cong Rong; Ge Gen; Tian Nan Xing; } \\
\text { Dan Shen; Shi Chang Pu; Jiang } \\
\text { Huang; Ba Ji Tian; Tian Ma; Chuan } \\
\text { Xiong. }\end{array}$ \\
\hline Pan et al., 2013 & $\begin{array}{l}\text { Yangxue Qingnao } \\
\text { granule }\end{array}$ & $\begin{array}{l}\text { Radix Angelicae Sinensis, Rhizoma } \\
\text { Ligustici Chuanxiong, Radix Paeoniae } \\
\text { Alba, Ramulus Uncariae Cum Uncis, } \\
\text { Caulis Spatholobi, Spica Prunellae, } \\
\text { Concha Margaritifera, Radix } \\
\text { Rehmanniae Recens, Semen } \\
\text { Cassiae, Rhizoma Corydalis, Herba } \\
\text { Asari. }\end{array}$ & $\begin{array}{l}\text { Chinese angelica, sichuan lovage } \\
\text { Rhizome, debark peony root, gambir } \\
\text { plant nod, suberect spatholobus } \\
\text { stem, common selfheal fruit-spike, } \\
\text { nacre, unprocessed rehmannia root, } \\
\text { cassia seed, yanhusuo, manchurian } \\
\text { wildginger. }\end{array}$ & $\begin{array}{l}\text { Dang Gui; Chuan Xiong; Bai shao; } \\
\text { Gou Teng; Ji Xue Teng; Xia Ku Cao; } \\
\text { Zhen Zhu Mu; Di Huang; Jue Ming Zi; } \\
\text { Yan Hu Suo; Xi Xin }\end{array}$ \\
\hline
\end{tabular}


TABLE 2 | Continued

\begin{tabular}{|c|c|c|c|c|}
\hline \multirow[t]{2}{*}{ Included studies } & \multirow[t]{2}{*}{ Prescription } & \multicolumn{3}{|c|}{ Ingredients } \\
\hline & & Latin name & English name & Chinese name \\
\hline Wen et al., 2015 & Congwu Xifeng granule & $\begin{array}{l}\text { Radix Polygoni Multiflori Preparata, } \\
\text { Herba Cistanches, Cortex } \\
\text { Eucommiae, Rhizoma Gastrodiae } \\
\text { Ramulus Uncariae Cum Uncis, } \\
\text { Rhizoma Atractylodis Macrocephalae, } \\
\text { Radix Paeoniae Alba, Rhizoma } \\
\text { Polygoni Cuspidati, Radix et Rhizoma } \\
\text { Rhei, }\end{array}$ & $\begin{array}{l}\text { Prepared fleeceflower root, } \\
\text { desertliving cistanche, eucommia } \\
\text { bark, tall gastrodia tuber, gambir plant } \\
\text { nod, largehead atractylodes rhizome, } \\
\text { debark peony root, giant knotweed } \\
\text { rhizome, rhubarb root and rhizome. }\end{array}$ & $\begin{array}{l}\text { Zhi He Shou Wu; Rou Cong Rong; Du } \\
\text { Zhong; Tian Ma, Gou Teng; Bai Zhu; } \\
\text { Bai Shao; Hu Zhang; Jiu Da Huang. }\end{array}$ \\
\hline Yang et al., 2017 & $\begin{array}{l}\text { Yishen Chuchan } \\
\text { decoction }\end{array}$ & $\begin{array}{l}\text { Radix Polygoni Multiflori } \\
\text { Preparata,Radix Rehmanniae } \\
\text { Recens, Rhizoma Gastrodiae, Radix } \\
\text { Paeoniae Alba, Concha Ostreae, } \\
\text { Bombyx Batryticatus, Radix et } \\
\text { Rhizoma Rhei, Radix Linderae, } \\
\text { Rhizoma Dioscoreae, Fructus Alpiniae } \\
\text { oxyphyllae. }\end{array}$ & $\begin{array}{l}\text { PREPARED fleeceflower root, } \\
\text { unprocessed rehmannia root, tall } \\
\text { gastrodia tuber, debark peony root, } \\
\text { oyster shell, stiff silkworm, rhubarb } \\
\text { root and rhizome, combined } \\
\text { spicebush root, common yam } \\
\text { rhizome, sharp-leaf glangal fruit. }\end{array}$ & $\begin{array}{l}\text { Zhi He Shou Wu, Sheng Di Huang, } \\
\text { Tian Ma, Bai Shao, Mu Li, Jiang Can, } \\
\text { Da Huang, wu Yao, Shan yao, Yi Zhi. }\end{array}$ \\
\hline Yu, 2016 & Naokang granule & $\begin{array}{l}\text { Herba Cistanches, Radix } \\
\text { Notoginseng, Rhizoma Ligustici } \\
\text { Chuanxiong, Radix Salviae } \\
\text { Miltiorrhizae, Rhizoma Acori } \\
\text { Tatarinowii, Radix Polygalae, } \\
\text { Scolopendra, Lumbricus, Bombyx } \\
\text { Batryticatus, Scorpio, Radix Astragali } \\
\text { seu Hedysari, Radix Codonopsis, } \\
\text { Herba Epimedii. }\end{array}$ & $\begin{array}{l}\text { Desertliving cistanche, sanqi, sichuan } \\
\text { lovage rhizome, danshen root, } \\
\text { grassleaf sweetflag rhizome, milkwort } \\
\text { root, centipede, earthworm, stiff } \\
\text { silkworm, scorpion, milkvetch root, } \\
\text { tangshen, epimedium herb. }\end{array}$ & $\begin{array}{l}\text { Rou Cong Rong, San Qi, Chuan } \\
\text { Xiong, Dan Shen, Shi Chang Pu, } \\
\text { Yuan Zhi, Wu Gong, Di Long, Jiang } \\
\text { Can, Quan Xie, Huang qi, Dang Shen, } \\
\text { Yin Yang Huo. }\end{array}$ \\
\hline $\begin{array}{l}\text { Zhao et al., 2009, } \\
2013\end{array}$ & $\begin{array}{l}\text { Guiling Pa'an capsule } \\
\text { Guiling Pa'an granule }\end{array}$ & $\begin{array}{l}\text { Carapax et Plastrum Testudinis, } \\
\text { Cornu Saigae Tataricae, et al. }\end{array}$ & $\begin{array}{l}\text { Tortoise carapace and plastron, } \\
\text { antelope horn, et al. }\end{array}$ & Gui Jia; Ling Yang Jiao, et al. \\
\hline
\end{tabular}

TABLE 3 | The 11 high-frequency used herbs for PD in the 14 trials included.

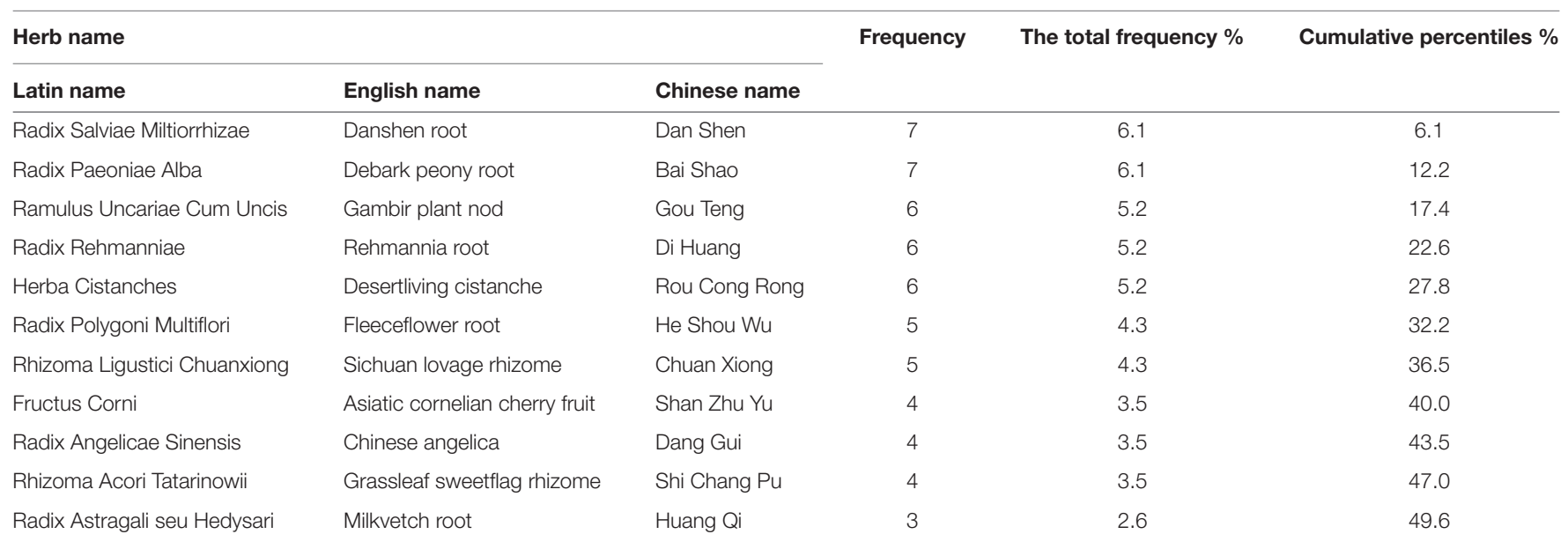

risks of bias were described in one study (Li et al., 2016), which reported significant differences in baseline values of some outcome variables.

\section{Effect Estimation}

\section{HM Monotherapy vs. Placebo}

One study (Zhao et al., 2009) showed that the efficacy of HM monotherapy was similar to placebo according to UPDRS II
$(P>0.05)$, UPDRS III $(P>0.05)$ and total UPDRS score $(P>0.05)$ in $\mathrm{PD}$ patients who never received levodopa treatment.

\section{HM Plus WCM vs. Placebo Plus WCM}

UPDRS I: Four studies (Pan et al., 2009, 2011; Chen M. Y. et al., 2014; Cai et al., 2017) with 5 comparisons showed that the HM paratherapy significantly improved UPDRS I compared with control groups (WMD: $-0.30,95 \% \mathrm{CI}:-0.54$ to $-0.06, P=0.02$; heterogeneity: $\mathrm{Chi}^{2}=3.21, P=0.52, I^{2}=0 \%$; Figure $3 \mathrm{~A}$ ). 

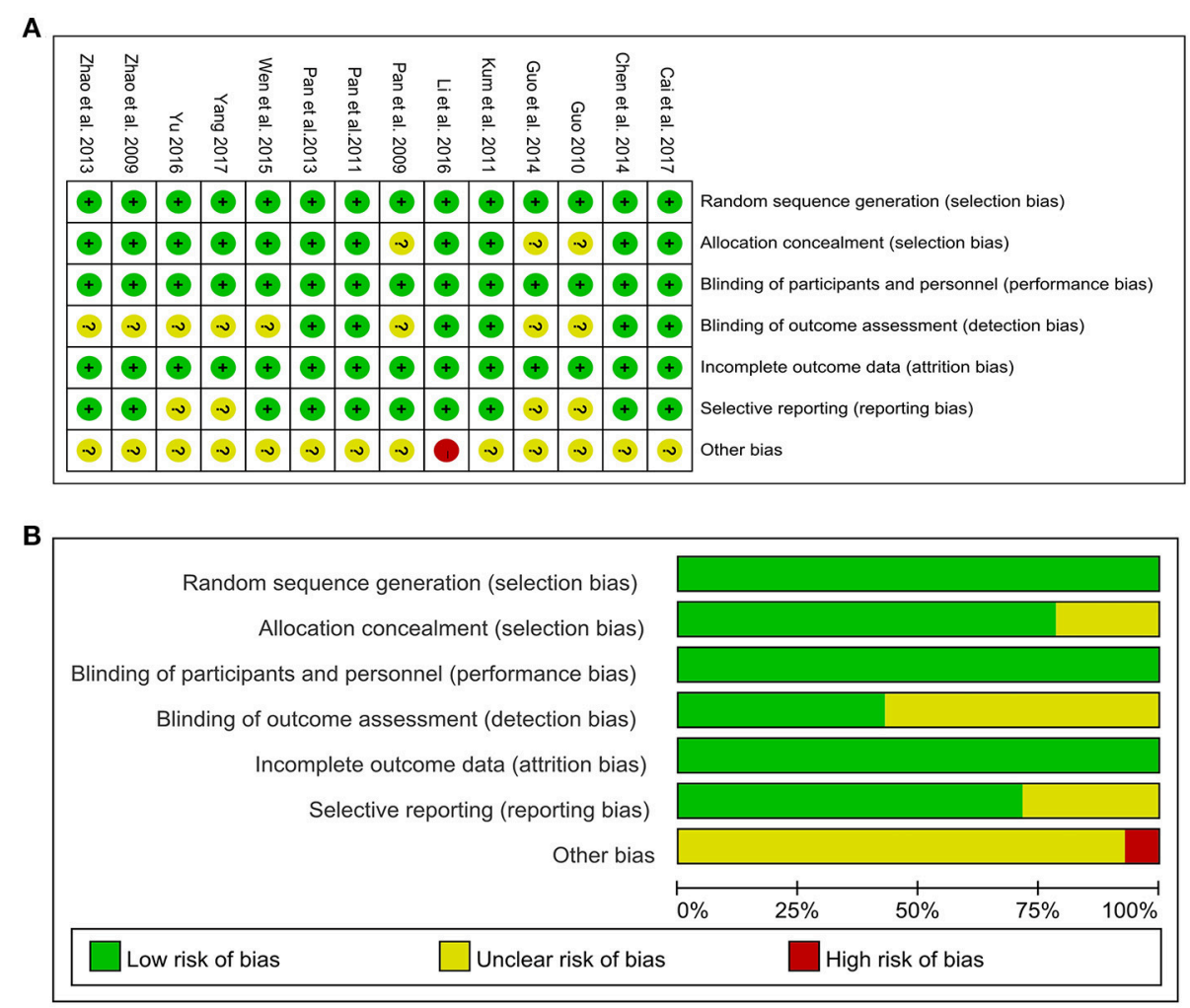

FIGURE 2 | Risk of bias of the included studies. (A) Risk of bias summary: judgements about each risk of bias item for each included study. (B) Risk of bias graph: judgements about each risk of bias item presented as percentages across all included studies. +, low risk of bias; -, high risk of bias; ?, unclear risk of bias.

UPDRS II: Seven studies (Pan et al., 2009, 2011; Zhao et al., 2009; Chen M. Y. et al., 2014; Li et al., 2016; Yu, 2016; Cai et al., 2017) with 9 comparisons assessed UPDRS II. Compared with the placebo, meta-analysis of 9 comparisons showed that HM paratherapy significantly improved UPDRS II (WMD: -1.53, 95\% CI -2.76 to $-0.30, P=0.01$; heterogeneity: $\mathrm{Chi}^{2}=19.42$, $\left.P=0.01, I^{2}=59 \%\right)$. Sensitivity analyses conducted to explore potential sources of heterogeneity. A trial (Li et al., 2016) had imbalanced baseline comparing HM with placebo. After removing the trial, meta-analysis of 8 comparisons (Pan et al., 2009, 2011; Zhao et al., 2009; Chen M. Y. et al., 2014; Yu, 2016; Cai et al., 2017) showed that HM paratherapy was still superior to the placebo (WMD: $-2.21,95 \% \mathrm{CI}:-3.19$ to $-1.22, P<0.0001$; heterogeneity: $\mathrm{Chi}^{2}=3.46, P=0.84, I^{2}=0 \%$; Figure $3 \mathrm{~B}$ ).

UPDRS III: Eight studies (Pan et al., 2009, 2011; Zhao et al., 2009; Chen M. Y. et al., 2014; Li et al., 2016; Yu, 2016; Cai et al., 2017; Yang, 2017) with 10 comparisons used UPDRS III as outcome measure. Compared with the placebo, meta-analysis of 10 comparisons showed that HM paratherapy had no significance for improving UPDRS III (WMD $=-2.13$, 95\% CI: -4.92 to $0.66, P=0.19$; heterogeneity: $\mathrm{Chi}^{2}=87.10, P<0.00001$, $\left.I^{2}=90 \%\right)$. A trial (Li et al., 2016) had imbalanced baseline comparing HM with placebo. After removing the trial, metaanalysis of 9 comparisons (Pan et al., 2009, 2011; Zhao et al., 2009; Chen M. Y. et al., 2014; Yu, 2016; Cai et al., 2017; Yang, 2017) showed that HM paratherapy was superior to the placebo (WMD:
$-3.26,95 \%$ CI: -4.36 to $-2.16, P<0.00001$; heterogeneity: $\mathrm{Chi}^{2}=1.88, P=0.98, I^{2}=0 \%$; Figure $\left.3 \mathrm{C}\right)$.

UPDRS IV: Meta-analysis of 5 studies (Pan et al., 2009, 2011; Kum et al., 2011; Chen M. Y. et al., 2014; Cai et al., 2017) with 6 comparisons revealed that HM paratherapy did not significantly improve UPDRS IV relative to placebo (WMD: $-0.18,95 \% \mathrm{CI}$ : -0.37 to $-0.01, P=0.06$; heterogeneity: $\mathrm{Chi}^{2}=5.76, P=0.33$, $I^{2}=13 \%$; Figure 3D).

Total UPDRS Score: Meta-analysis of 4 studies (Pan et al., 2009, 2011; Zhao et al., 2009; Chen M. Y. et al., 2014) with 6 comparisons revealed that HM paratherapy significantly improved the total UPDRS scores relative to placebo (WMD: $-5.43,95 \% \mathrm{CI}:-8.01$ to $-2.86, P<$ 0.0001; heterogeneity: $\mathrm{Chi}^{2}=2.59, P=0.76, I^{2}=0 \%$; Figure 3E).

Quality of Life: Compared with the placebo, meta-analysis of 4 studies (Kum et al., 2011; Wen et al., 2015; Li et al., 2016; Yang, 2017) showed that HM paratherapy had no significance for improving PDQ-39 (WMD: $-4.65,95 \% \mathrm{CI}$ : -10.97 to 1.68 , $P=0.15$; heterogeneity: $\mathrm{Chi}^{2}=19.19, P=0.0002, I^{2}=84 \%$ ). A trial (Li et al., 2016) had imbalanced baseline comparing HM with placebo. After removing the trial, meta-analysis of 3 studies (Kum et al., 2011; Wen et al., 2015; Yang, 2017) showed that HM paratherapy was superior to the placebo (WMD: $-7.65,95 \% \mathrm{CI}:-11.46$ to $-3.83, p<0.0001$; heterogeneity: $\mathrm{Chi}^{2}=0.12, P=0.94, I^{2}=0 \%$; Figure 4A). One randomized 
controlled trial (RCT) (Cai et al., 2017) showed HM paratherapy was a significant superiority to placebo according to PDQL $(P<0.01)$.

NMSQuest and NMSS: One trial (Cai et al., 2017) showed that HM paratherapy produced greater reduction in NMSQuest score than that of placebo $(P<0.01)$. Meta-analysis of 2 studies (Yu, 2016; Yang, 2017) showed that HM paratherapy was favor of NMSS compared with placebo (WMD: $-9.19,95 \%$ CI: -13.11 to $-5.28, P<0.00001$; heterogeneity: $\mathrm{Chi}^{2}=0.56, P=0.45$, $I^{2}=0 \%$; Figure $4 \mathrm{~B}$ ).

PDSS: Compared with the placebo, meta-analysis of 3 studies (Pan et al., 2013; Li et al., 2016; Cai et al., 2017) showed that HM paratherapy had no significance for improving PDSS (WMD: $7.10,95 \% \mathrm{CI}:-2.26$ to $16.45, P=0.14$; heterogeneity: $\mathrm{Chi}^{2}=101.02, P=0.14, I^{2}=98 \%$ ). A trial (Li et al., 2016) had imbalanced baseline comparing HM with placebo. After removing the trial, meta-analysis of 2 studies (Pan et al., 2013; Cai et al., 2017) showed that HM paratherapy was superior to the placebo (WMD: $10.69,95 \% \mathrm{CI}: 8.86$ to $12.53, P<$ 0.00001; heterogeneity: $\mathrm{Chi}^{2}=0.48, P=0.49, I^{2}=0 \%$; Figure 4C).

HAMD: Meta-analysis of 2 studies (Guo et al., 2014; Cai et al., 2017) showed that HM paratherapy was superior to the placebo according to HAMD (WMD: $-5.87,95 \% \mathrm{CI}:-7.06$ to -4.68 , $p<0.00001)$ with mild heterogeneity $\left(\mathrm{Chi}^{2}=1.51, P=0.22\right.$, $I^{2}=34 \%$; Figure 4D).

\section{Adverse Events}

\section{HM Monotherapy vs. Placebo}

In the only one study (Zhao et al., 2009), neither the experimental group nor the control group provide any information about adverse events.

\section{HM Plus WCM vs. Placebo Plus WCM}

Eleven RCTs (Guo, 2010; Kum et al., 2011; Pan et al., 2011, 2013; Zhao et al., 2013; Chen M. Y. et al., 2014; Guo et al., 2014; Li et al., 2016; Yu, 2016; Cai et al., 2017; Yang, 2017) reported adverse events, among them 3 studies (Pan et al., 2011, 2013; Guo et al., 2014) reported no adverse events. However, the other 3 studies did not provide any information on adverse event (Pan et al., 2009; Zhao et al., 2009; Wen et al., 2015). Meta-analysis of 8 studies (Guo, 2010; Kum et al., 2011; Zhao et al., 2013; Chen M. Y. et al., 2014; Li et al., 2016; Yu, 2016; Cai et al., 2017; Yang, 2017) showed that HM paratherapy was significant benefit in reducing adverse events compared with control group (RR: $0.41,95 \%$ CI: 0.21 to $0.80, P=0.009$; heterogeneity: $\mathrm{Chi}^{2}=$ 6.36, $P=0.38, I^{2}=6 \%$; Figure 5). The most reported adverse events were gastrointestinal symptoms, such as nausea, vomiting, diarrhea, and abdominal distention in both the HM groups and placebo groups. No life-threatening adverse event was noted in all studies.

\section{Publication Bias}

We did not performed the Funnel plot and Egger's test because the number of studies in each meta-analysis was less than ten.

\section{DISCUSSION}

\section{Summary of Evidence}

This is first systematic review of randomized double-blind placebo-controlled clinical trials to assess the efficacy and safety of HM formulas for PD. Fourteen high-quality randomized controlled trials (Pan et al., 2009, 2011, 2013; Zhao et al., 2009, 2013; Guo, 2010; Kum et al., 2011; Chen M. Y. et al., 2014; Guo et al., 2014; Wen et al., 2015; Li et al., 2016; Yu, 2016; Cai et al., 2017; Yang, 2017) involving 1,316 patients suffering from PD were identified. HM paratherapy was significant for improving motor symptoms and non-motor functions, whereas there was a negative result of complications of treatment. One trail (Zhao et al., 2009) indicated that HM monotherapy was not superior to the placebo. Eleven out of fourteen studies (Guo, 2010; Kum et al., 2011; Pan et al., 2011, 2013; Zhao et al., 2013; Chen M. Y. et al., 2014; Guo et al., 2014; Li et al., 2016; Yu, 2016; Cai et al., 2017; Yang, 2017) reported no serious adverse events relevant with HM formulas, indicating that HM formulas were generally safe and well tolerated for PD patients. Thus, the findings of present study supported the complementary use of HM paratherapy for PD patients, whereas HM monotherapy for PD is still lack of evidence.

\section{LIMITATIONS}

First, the members of the International Committee of Medical Journal Editors published a statement requiring that all clinical trials must be registered in order to be considered for publication (DeAngelis et al., 2004). However, most of included studies didn't formally register. Protocols were not available to confirm free of selective reporting. Thus, further clinical trials must register prospectively in international clinical trials registry platform. Second, although we included randomized double-blind placebocontrolled trials, some inherent and methodological weaknesses still existed in the primary studies: (1) An adequate sample size is crucial to the design of RCTs (Lewis, 1999), but only 4 trials (Kum et al., 2011; Zhao et al., 2013; Li et al., 2016; $\mathrm{Yu}, 2016$ ) applied pre-trial sample size estimation; (2) PD is a chronic degenerative disease. Long-term efficacy and safety are important assessments to decide the clinical usefulness of an agent in treatment, but only one trial (Li et al., 2016) had the long-term duration of follow-up at 6 month; (3) Intention-totreat (ITT) analysis could avoid bias and false-positive results, which is the recommended standard approach to analyse data from RCTs (Abraha et al., 2017). However, only two studies (Kum et al., 2011; Li et al., 2016) adopted ITT analysis. (4) In the present study, only 6 trials conducted assessor blinding. Considering the characteristics of outcome measurement of PD patients (e.g., UPDRS), assessor blinding successfully eliminates assessment bias and increases the accuracy and objectivity of outcomes results. Triple blindness is needed in further PD trials. Thus, CONSORT 2010 statement (Moher et al., 2010a) and CONSORT Extension for Chinese Herbal Medicine Formulas 2017 (Cheng et al., 2017) should be applied in trial reporting and publication. Third, the herbal composition, drug formulation 


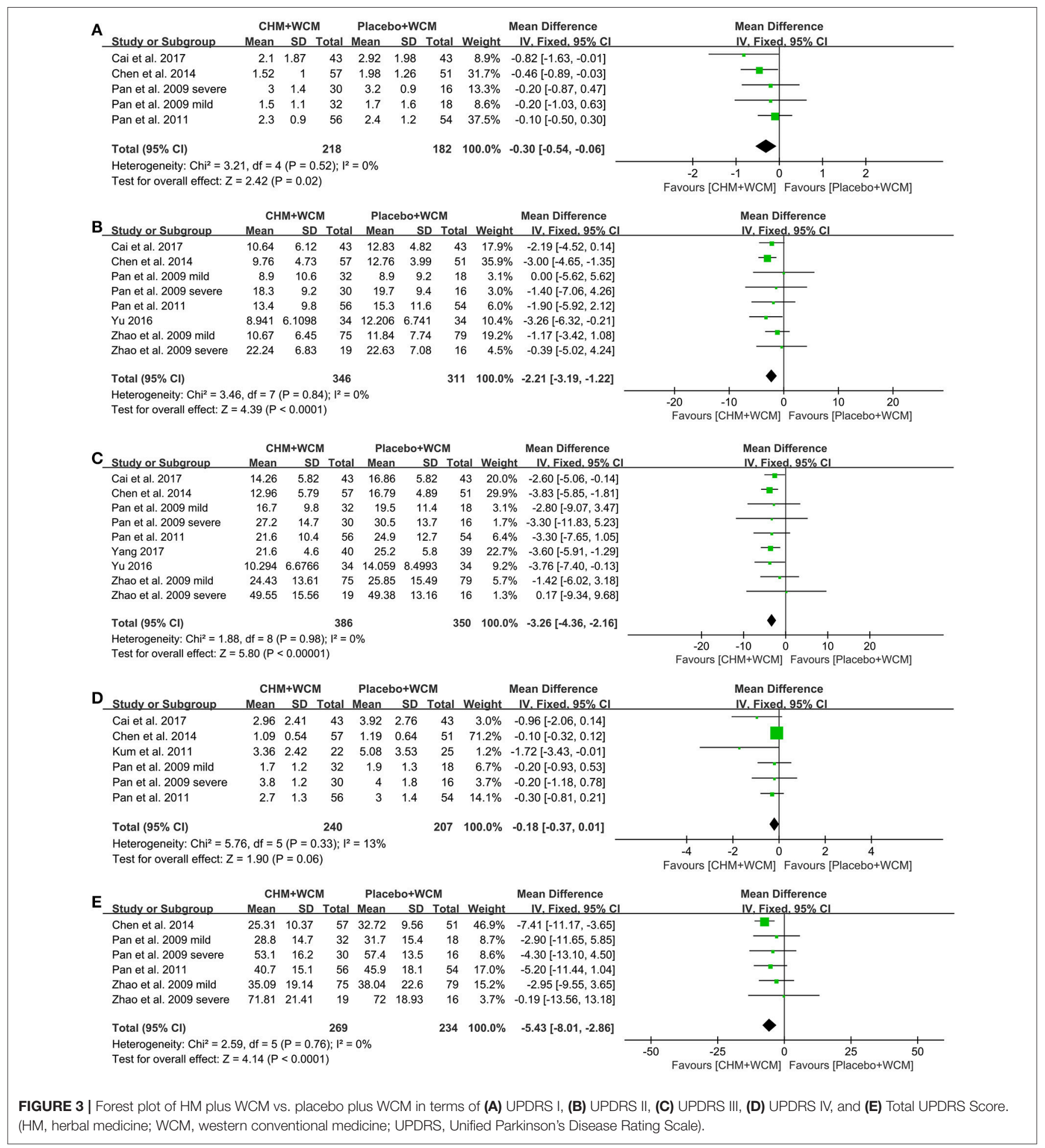

and dose of the intervention were not exact same, which would lead to clinical heterogeneity. To assess the efficacy and safety of HMs in a clinical trial, all subjects should be given exactly the same intervention in terms of product identity, purity, dosage, and formulation. Fourth, our study only included trials published in the English and Chinese languages and all the included studies were conducted in China, which may affect the generalizability of present findings. In the further studies, the international coliaboration is needed in order to get more qualified stuidies. Finally, different types and stages of PD can influence disease progression and response to treatment (Reinoso et al., 2015). It is difficult to differentiate the effectiveness of 


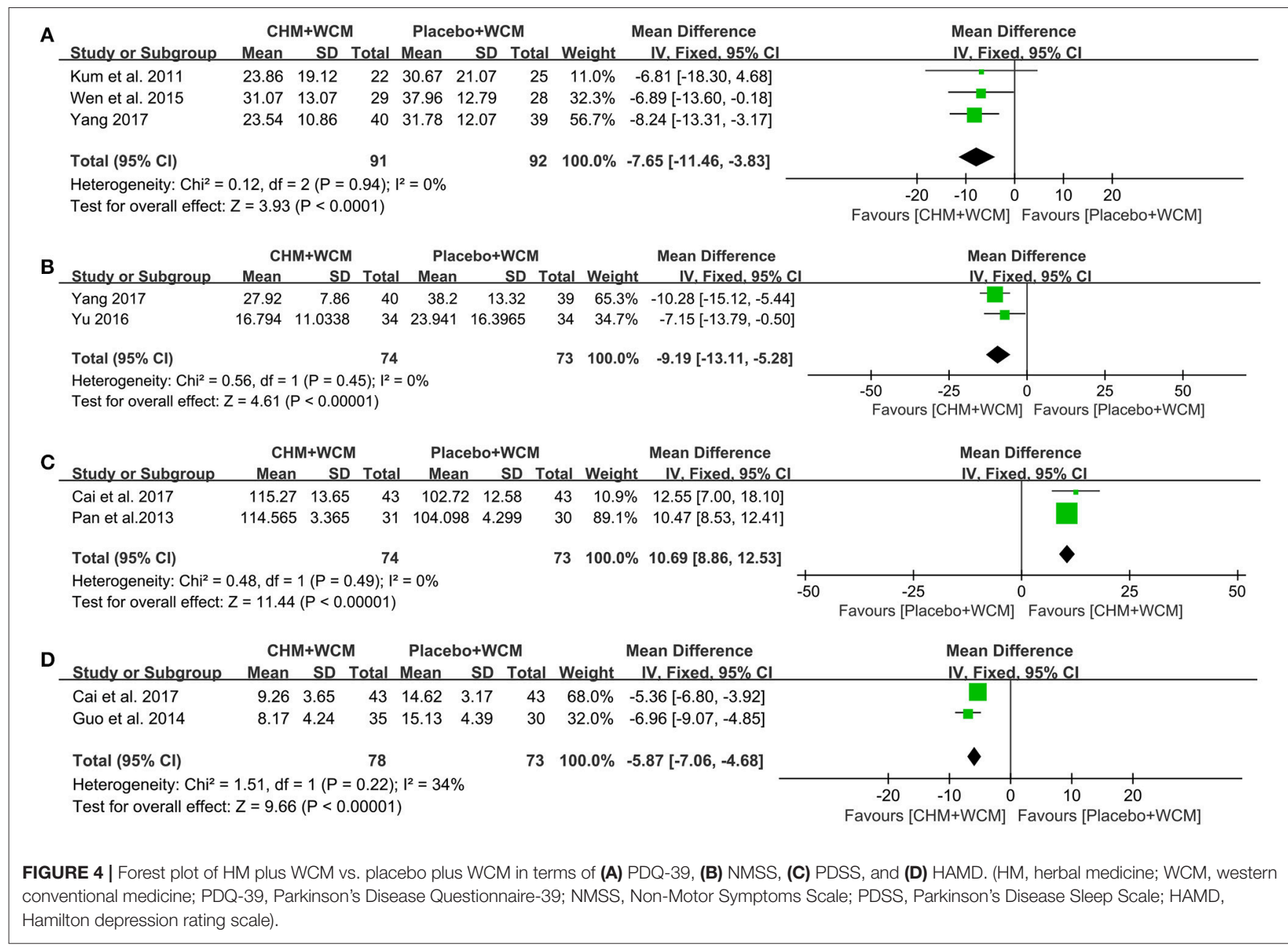

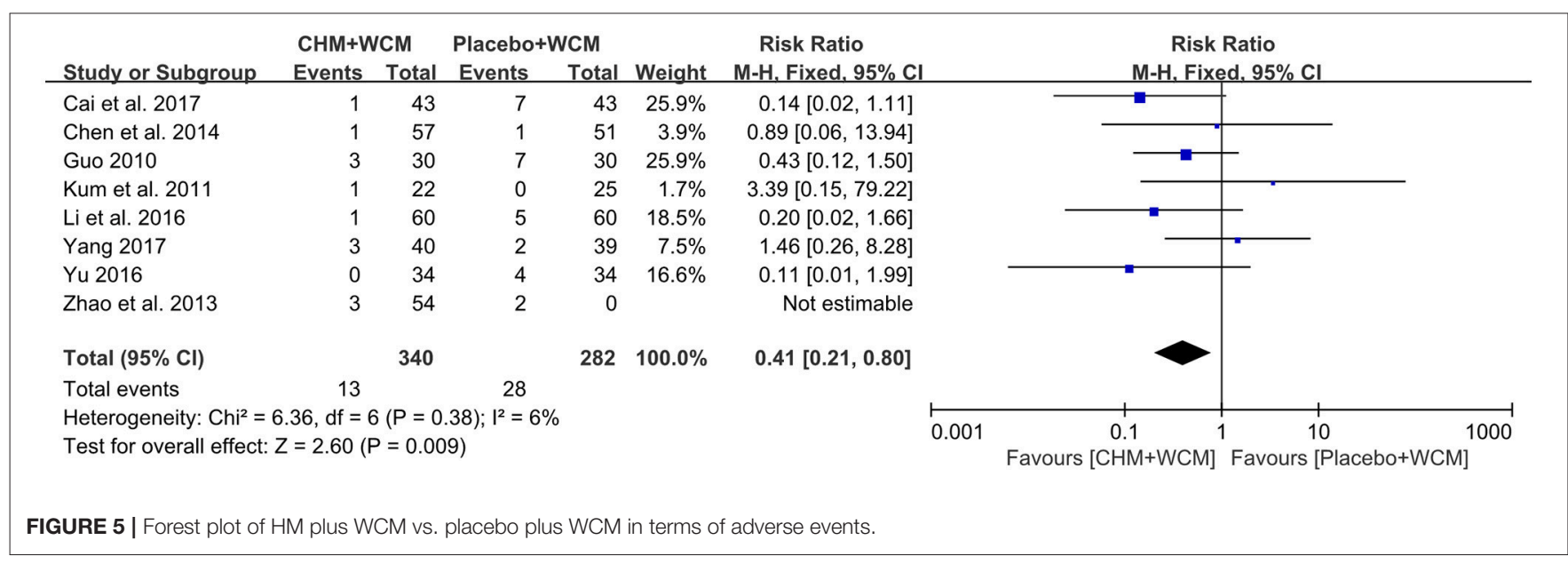

HM formulas targeting these subgroups due to insufficient data of primary studies. The pertinent research should be conducted in future clinical trials, which would contribute significantly to explore the responsiveness of specific PD subgroup to interventions.

\section{Implications}

Up to now, several systematic reviews of traditional medicine for PD (Kim et al., 2012; Wang et al., 2012; Zhang et al., 2014, 2015) have been performed. However, low-quality of included primary studies hindered our conclusions. For example, the 
two articles written by Kim et al. (2012) and Zhang et al. (2015) belong to high-quality systematic reviews; however, the inherent limitations existed in the included low-quality primary studies. The present study only included randomized double-blind placebo-controlled trials, which remains the gold standard of trial design (Athauda and Foltynie, 2016). These trials reported the detailed randomized methods; placebocontrolled group accounts for the placebo effects that don't depend on the treatment itself (Chen et al., 2017), and thus increasing the reliability of experiment results. The present study provided the evidence to support HM paratherapy for $\mathrm{PD}$, whereas there is still lack of available evidence for HM monotherapy for PD. However, it should be remembered that a lack of scientific evidence does not necessarily mean that the treatment is ineffective (Kotsirilos, 2005). To explore the efficacy of HM monotherapy for PD is needed in the future.

Currently, most available PD therapies are mainly aimed at motor symptoms (Fox et al., 2018). Non-motor symptoms (NMS) are common in PD patients across all disease stages and are a key determinant of QOL (Martinez-Martin et al., 2012). However, NMS have received limited attention and targeted treatments remain a challenge (Kulisevsky et al., 2018). The present systematic review provided the sportive evidence for the effectiveness and safety of HM paratherapy for NMS of PD patients. Thus, it is worthy of further studies.

Although the exact pathogenic mechanisms underlying selective dopaminergic neurons loss in PD remain unknown, it is believed that oxidative stress and mitochondrial dysfunction, protein misfolding and aggregation, inflammation, and apoptotic cell death play central roles in PD pathogenesis (Sarkar et al., 2016). Obviously, PD is not a result of dysfunction of one specific pathway but rather a combination of interconnected events (Lim and Zhang, 2013). The urgent need in PD is the development of neuroprotective therapy targeting more potential signal pathways (Kalia et al., 2015). However, clinical neuroprotective effects of current agents in PD remain unproven (Löhle and Reichmann, 2010). The most frequently used herbs of HM formulas were selected in the present study, including Radix Salviae Miltiorrhizae, Radix Paeoniae Alba, Ramulus Uncariae Cum Uncis, Radix Rehmanniae, Herba Cistanches, Radix Polygoni Multiflori, Rhizoma Ligustici Chuanxiong, Fructus Corni, Radix Angelicae Sinensis, Rhizoma Acori Tatarinowii, and Radix Astragali seu Hedysari. Based on the high-frequency used herbs, the anti-PD mechanisms of the main active ingredients of herbs in vivo or in vitro trails are as follows: (1) Antioxidant: Danshensu (from Radix Salviae Miltiorrhizae), catalpol (from Radix Rehmanniae), 2,3,5,4'-Tetrahydroxystilbene-2-O- $\beta$-D-Glucoside (TSG) (from Radix Polygoni Multiflori), morroniside (from Fructus Corni) and astragaloside IV (AS-IV) (from Radix Astragali seu Hedysari) were shown to alleviate oxidative stress through reducing reactive oxygen species (ROS) level (Bi et al., 2008a; Sun et al., 2011; Chong et al., 2013; Liu et al., 2017; Zhang et al., 2017). Catalpol, and tetramethylpyrazine (TMP) (from Rhizoma Ligustici Chuanxiong) prevented the decrease in the activities of superoxide dismutase, catalase and glutathione peroxidase, and inhibited malondialdehyde overproduction (Bi et al., 2008a; Lu et al., 2014; Li et al., 2016). The Regulation of I3K/Akt/Nrf2 signaling pathway by Danshensu (Chong et al., 2013) and the inhibition of Nrf2/HO-1 pathway by TMP (Michel et al., 2017) contributed to their antioxidant role; (2) Antiinflammatory: Echinacoside (ECH) (from Herba Cistanches) and catalpol showed a stronger inhibition on the productions and/or expressions of several pro-inflammatory cytokine, including nitric oxide (Tian et al., 2006), tumor necrosis factor- $\alpha$ (Tian et al., 2006), interleukin (IL)-1 $\alpha$ (Tian et al., 2006), IL-1 $\beta$ and IL-6 (Wang et al., 2015). TMP (Michel et al., 2017) may inhibit the expression of neuroinflammation markers: nuclear factor $\kappa \mathrm{B}(\mathrm{NF}-\kappa \mathrm{B})$, inducible nitric oxide synthase, cyclooxygenase2, and glial fibrillary acidic protein; (3) Anti-apoptotic: ECH, TSG, morroniside, paeoniflorin (PF) (from Radix Paeoniae Alba), TMP, n-Butylidenephthalide (BP) (from Radix Angelicae Sinensis), or AS-IV exerted anti-apoptotic capacity in different aspects, including suppressing the upregulation of the ratio of Bax/Bcl-2 (Sun et al., 2012; Lu et al., 2014; Liu et al., 2017; Michel et al., 2017), the activation of caspase-3 and caspase8 (Geng et al., 2007; Sun et al., 2011; Lu et al., 2014; Michel et al., 2017) and the expression of Proapoptotic Gene egl-1 (Fu et al., 2014). TSG (Qin et al., 2011) reduced MPP+-induced apoptotic that mediated via PI3K/Akt signaling pathway; (4) The Regulation of mitochondrial dysfunction: ECH, TSG, PF or catalpol attenuated mitochondrial dysfunction not only by suppressing the decrease of cellular ATP levels (Wang et al., 2015), mitochondrial membrane potential (Bi et al., 2008b; Sun et al., 2011, 2012; Wang et al., 2015), the activity of mitochondrial complex I (Bi et al., 2008b), but also decreasing mitochondrial permeability transition pore opening (Bi et al., 2008b); (5) DA and dopaminergic neuron protection: Danshensu, ECH, BP, TMP, AS- IV or $\beta$-asarone (from Rhizoma Acori Tatarinowii) could enhance the content of DA as well as its metabolites and reduce dopaminergic neuron degeneration (Geng et al., 2007; Chong et al., 2013; Fu et al., 2014; Lu et al., 2014), and led to a marked increase in Tyrosine hydroxylase expression (Geng et al., 2007; Lu et al., 2014; Zhang et al., 2016; Liu et al., 2017). Furthermore, catalpol (Bi et al., 2008b) was found to be a strong inhibitor of MAO-B,which may weaken the biotransformation of 1-methyl-4-phenyl-1,2,3,6-tetrahydropyridine to 1-methyl4-phenylpyridinium and the metabolism of DA; (6) Reduce $\alpha$-synuclein accumulation: $\alpha$-synuclein $(\alpha$-syn) is a major component of lewy bodies that plays an important role in the pathogenesis of PD (Rocha et al., 2018). Corynoxine (Chen L. L. et al., 2014) (from Ramulus Uncariae Cum Uncis) down regulated $\alpha$-syn in PC12 cells by inducing autophagy. AS-IV (Liu et al., 2017) inhibited the expression of the $\alpha$-syn via the p38 MAPK signaling pathway. Furthermore, $\beta$-asarone (Zhang et al., 2016) promoted the clearance of $\alpha$-syn via regulating long non-coding RNA Metastasis associated lung adenocarcinoma transcript 1. Because of their advantage of multi-component, multitarget and multi-pathway, HM formulas have great potential application value in neuroprotection. Furthermore, based on the high-frequency used herbs, we can explore the best formula combination, which also ignite the HM treatment method for PD patients. 


\section{CONCLUSION}

The findings of present study showed that HM paratherapy can effectively improve the motor symptoms and non motor symptoms of PD and is well tolerated for PD patients. Thus, the available evidence supported the complementary use of HM paratherapy for PD patients; however, the question on the efficacy of HM monotherapy in alleviating PD symptoms is still open.

\section{AUTHOR CONTRIBUTIONS}

G-QZ and C-SS: study conception and design; C-SS, H-FZ, Q-QX, Y-HS, YW, YLi, YLin, and G-QZ: acquisition,

\section{REFERENCES}

Abraha, I., Cozzolino, F., Orso, M., Marchesi, M., Germani, A., Lombardo, G., et al. (2017). A systematic review found that deviations from intention-to-treat are common in randomized trials and systematic reviews. J. Clin. Epidemiol. 84, 37-46. doi: 10.1016/j.jclinepi.2016.11.012

Athauda, D., and Foltynie, T. (2015). The ongoing pursuit of neuroprotective therapies in Parkinson disease. Nat. Rev. Neurol. 11, 25-40. doi: 10.1038/nrneurol.2014.226

Athauda, D., and Foltynie, T. (2016). Challenges in detecting disease modification in Parkinson's disease clinical trials. Parkinson. Relat. Disord. 32, 1-11. doi: 10.1016/j.parkreldis.2016.07.019

Bi, J., Jiang, B., Liu, J. H., Lei, C., Zhang, X. L., and An, L. J. (2008a). Protective effects of catalpol against $\mathrm{H} 2 \mathrm{O} 2$-induced oxidative stress in astrocytes primary cultures. Neurosci Lett. 442, 224-227. doi: 10.1016/j.neulet.2008.07.029

Bi, J., Wang, X.B., Chen, L., Hao, S., An, L.J., Jiang, B., et al. (2008b). Catalpol protects mesencephalic neurons against MPTP induced neurotoxicity via attenuation of mitochondrial dysfunction and MAO-B activity. Toxicol in vitro 22, 1883-1889. doi: 10.1016/j.tiv.2008.09.007

Cai, L., Liu, Y., and Li, W.T. (2017). Combination of Zhichan decoction and western medicine for the treatment of 43 cases of Parkinson's disease: a randomized double-blind controlled trial. Jiangsu J. Tradit. Chin. Med. 49, 33-35. doi: 10.3969/j.issn.1672-397X.2017.11.013

Chen, L. L., Song, J. X., Lu, J. H., Yuan, Z. W., Liu, L. F., Durairajan, S. S., et al. (2014). Corynoxine, a natural autophagy enhancer, promotes the clearance of alpha-synuclein via Akt/mTOR pathway. J. Neuroimmune Pharmacol. 9, 380-387. doi: 10.1007/s11481-014-9528-2

Chen, M. Y., Liu, Y., Ruan, K. F., Zhang, J. Q., Hong, Y. L., Zhu, J. Y., et al. (2014). Combination of Zhichan granules and western medicine for the treatment of Parkinson's disease of liver kidney deficiency pattern and qi blood deficiency pattern: a randomized double-blind placebo-controlled clinical trial. Shanghai J. Tradit. Chin. Med. 28, 27-30. doi: 10.16305/j.1007-1334.2014.10.034

Chen, Z. X., Li, Y., Zhang, X. G., Chen, S., Yang, W. T., Zheng, X. W., et al. (2017). Sham electroacupuncture methods in randomized controlled trials. Sci. Rep. 7:40837. doi: 10.1038/srep40837

Cheng, C.W., Wu, T.X., Shang, H.C., Li, Y.P., Altman, D.G., Moher, D., et al. (2017). CONSORT extension for Chinese herbal medicine formulas 2017: recommendations, explanation, and elaboration (simplified Chinese version). Ann. Intern. Med. 167, W21-W34. doi: 10.7326/M16-2977

Chong, C. M., Zhou, Z. Y., Razmovski-Naumovski, V., Cui, G. Z., Zhang, L. Q., Sa, F., et al. (2013). Danshensu protects against 6-hydroxydopamine-induced damage of PC12 cells in vitro and dopaminergic neurons in zebrafish. Neurosci. Lett. 543, 121-125. doi: 10.1016/j.neulet.2013.02.069

DeAngelis, C., Drazen, J. M., Frizelle, F. A., Haug, C., Hoey, J., Horton, R., et al. (2004). Clinical trial registration: a statementfrom the International committee of medical journal editors. Med. J. Aust. 181, 293-294. doi: $10.1001 /$ jama.292.11.1363

Findley, L.J., Wood, E., Lowin, J., Roeder, C., Bergman, A., and Schifflers, M. (2011). The economic burden of advanced Parkinson's disease: analysis and/or interpretation of data; G-QZ: final approval and overall responsibility for this published work.

\section{FUNDING}

This work was financially supported by the grant of National Natural Science Foundation of China (81573750/81473491/81173395/H2902); the Young and Middle-Aged University Discipline Leaders of Zhejiang Province, China (2013277); Zhejiang Provincial Program for the Cultivation of High-level Health talents (2015). an analysis of a UK patient dataset. J. Med. Econ. 14, 130-139. doi: 10.3111/13696998.2010.551164

Fox, S. H., Katzenschlager, R., Lim, S. Y., Barton, B., de Bie, R. M. A., Seppi, K., et al. (2018). International Parkinson and movement disorder society evidencebased medicine review: update on treatments for the motor symptoms of Parkinson's disease. Mov. Disord. 33, 1248-1266. doi: 10.1002/mds.27372

Fu, R. H., Harn, H. J., Liu, S. P., Chen, C. S., Chang, W. L., Chen, Y. M., et al. (2014). $\mathrm{n}$-butylidenephthalide protects against dopaminergic neuron degeneration and $\alpha$-synuclein accumulation in Caenorhabditis elegans models of Parkinson's disease. PLoS ONE 9:e85305. doi: 10.1371/journal.pone.0085305

GBD, 2015 Mortality and Causes of Death Collaborators (2016). Global, regional, and national life expectancy, all-cause mortality, and causespecific mortality for 249 causes of death, 1980-2015: a systematic analysis for the Global Burden of Disease Study 2015. Lancet 388, 1459-1544. doi: 10.1016/S0140-6736(16)31012-1

GBD, 2016 Disease and Injury Incidence and Prevalence Collaborators (2017). Global, regional, and national incidence, prevalence, and years lived with disability for 328 diseases and injuries for 195 countries, 1990-2016: a systematic analysis for the Global Burden of Disease Study 2016. Lancet 390, 1211-1259. doi: 10.1016/S0140-6736(17)32154-2

Geng, X., Tian, X., Tu, P., and Pu, X. (2007). Neuroprotective effects of echinacoside in the mouse MPTP model of Parkinson's disease. Eur. J. Pharmacol. 564, 66-74. doi: 10.1016/j.ejphar.2007. 01.084

Guo, C.H. (2010). Observation of Clinical Curation Effect on Gui Lu Di Huang Capsule for the Treatment of Parkinson's Disease Which is Liver-Kidney yin Deficiency Type. Dissertation, Guangzhou: Guangzhou University of Chinese Medicine (in Chinese).

Guo, Y. X., Li, S. D., and Yang, M. H. (2014). Bushen Huoxue Granule (BSHXG) for clinical studies of Parkinson's disease with depression. Glob. Traditional Chinese Med. 7, 275-278. doi: 10.3969/j.issn.1674-1749.2014.04.009

Higgins, J. P., Altman, D. G., Gøtzsche, P. C., Jüni, P., Moher, D., Oxman, A. D., et al. (2011). The Cochrane collaboration's tool for assessing risk of bias in randomised trials. BMJ 343:d5928. doi: 10.1136/bmj.d5928

Hughes, A. J., Daniel, S. E., Kilford, L., and Lees, A. J. (1992). Accuracy of clinical diagnosis of idiopathic Parkinson's disease: a clinico-pathological study of 100 cases. J. Neurol. Neurosurg. Psychiatry 55, 181-184. doi: 10.1136/jnnp.55.3.181

Jenner, P. (2015). Treatment of the later stages of Parkinson's disease pharmacological approaches now and in the future. Transl. Neurodegener. 4:3. doi: 10.1186/2047-9158-4-3

Kalia, L. V., Kalia, S. K., and Lang, A. E. (2015). Disease-modifying strategies for Parkinson's disease. Mov. Disord. 30, 1442-1450. doi: 10.1002/mds.26354

Kalia, L. V., and Lang, A. E. (2015). Parkinson's disease. Lancet 386, 896-912. doi: 10.1016/S0140-6736(14)61393-3

Kim, T. H., Cho, K. H., Jung, W. S., and Lee, M. S. (2012). Herbal medicines for Parkinson's disease: a systematic review of randomized controlled trials. PLoS ONE 7:e35695. doi: 10.1371/journal.pone.0035695

Kotsirilos, V. (2005). Complementary and alternative medicine. Part 2evidence and implications for GPs. Aust. Fam. Physician 34, 689-691. 
Kowal, S. L., Dall, T. M., Chakrabarti, R., Storm, M. V., and Jain, A. (2013). The current and projected economic burden of Parkinson's disease in the United States. Mov. Disord. 28, 311-318. doi: 10.1002/mds.25292

Kulisevsky, J., Oliveira, L., and Fox, S. H. (2018). Update in therapeutic strategies for Parkinson's disease. Curr. Opin. Neurol. 31, 439-47. doi: 10.1097/WCO.0000000000000579

Kum, W. F., Durairajan, S. S., Bian, Z. X., Man, S. C., Lam, Y. C., Xie, L. X., et al. (2011). Treatment of idiopathic Parkinson's disease with traditional chinese herbal medicine: a randomized placebo-controlled pilot clinical study. Evid. Based Complement. Alternat. Med. 2011:724353. doi: 10.1093/ecam/nep116

Lewis, J. A. (1999). Statistical principles for clinical trials (ICH E9): an introductory note on an international guideline. Stat Med. 18, 1903-1942. doi: 10.1002/ (SICI)1097-0258(19990815)18:15\&lt;1903::AID-SIM188\&gt;3.0.CO;2-F

Li, M., Yang, H. M., Luo, D. X., Chen, J. Z., and Shi, H. J. (2016). Multidimensional analysis on Parkinson's disease questionnaire-39 in Parkinson's patients treated with Bushen Huoxue Granule: a multicenter, randomized, double-blinded and placebo controlled trial. Complement Ther. Med. 29, 116-120. doi: 10.1016/j.ctim.2016.09.008

Lim, K. L., and Zhang, C. W. (2013). Molecular events underlying Parkinson's disease-an interwoven tapestry. Front. Neurol. 4:33. doi: 10.3389/fneur.2013.00033

Liu, X., Zhang, J., Wang, S., Qiu, J., and Yu, C. (2017). Astragaloside IV attenuates the $\mathrm{H} 2 \mathrm{O} 2$-induced apoptosis of neuronal cells by inhibiting $\alpha$-synuclein expression via the p38 MAPK pathway. Int. J. Mol. Med. 40, 1772-1780. doi: 10.3892/ijmm.2017.3157

Löhle, M., and Reichmann, H. (2010). Clinical neuroprotection in Parkinson's disease-still waiting for the breakthrough. J. Neurol. Sci. 289, 104-114. doi: 10.1016/j.jns.2009.08.025

Lu, C., Zhang, J., Shi, X., Miao, S., Bi, L., Zhang, S., et al. (2014). Neuroprotective effects of tetramethylpyrazine against dopaminergic neuron injury in a rat model of Parkinson's disease induced by MPTP. Int. J. Biol. Sci. 10, 350-357. doi: 10.7150/ijbs.8366

Martinez-Martin, P., Falup Pecurariu, C., Odin, P., van Hilten, J. J., Antonini, A.,Rojo-Abuin, J. M., et al. (2012). Gender-related differences in the burden of non-motor symptoms in Parkinson's disease. J. Neurol. 259, 1639-1647. doi: 10.1007/s00415-011-6392-3

Michel, H. E., Tadros, M. G., Esmat, A., Khalifa, A. E., and Abdel-Tawab, A. M. (2017). Tetramethylpyrazine ameliorates rotenone-induced parkinson's disease in rats: involvement of its anti-inflammatory and anti-apoptotic actions. Mol. Neurobiol. 54, 4866-4878. doi: 10.1007/s12035-016-0028-7

Moher, D., Hopewell, S., Schulz, K. F., Montori, V., Gøtzsche, P. C., Devereaux, P. J., et al. (2010a). CONSORT 2010 explanation and elaboration: updated guidelines for reporting parallel group randomised trials. J. Clin. Epidemiol. 63, e1-e37. doi: 10.1136/bmj.c869

Moher, D., Liberati, A., Tetzlaff, J., Altman, D. G., and PRISMA Group (2010b). Preferred reporting items for systematic reviews and meta-analyses: the PRISMA statement. Int. J. Surg. 8, 336-341. doi: 10.1016/j.ijsu.2010.02.007

Pan, W., Kwak, S., Li, G., Chen, Y., and Cai, D. (2013). Therapeutic effect of YangXue-Qing-Nao granules on sleep dysfunction in Parkinson's disease. Chin Med. 8:14. doi: 10.1186/1749-8546-8-14

Pan, W., Kwak, S., Liu, Y., Sun, Y., Fang, Z., Qin, B., et al. (2011). Traditional chinese medicine improves activities of daily living in Parkinson's disease. Parkinsons Dis. 2011:789506. doi: 10.4061/2011/789506

Pan, W. D., Yuan, W. A., Fang, Z. L., Liu, Y., and Guo, S. (2009). Effects of Zengxiao Anshen Zhichan 2 Decoction on motor and non-motor symptoms in Parkinson's disease. Acta Univ. Tradit. Med. Sin. Pharmacol. 23, 29-34. doi: 10.16306/j.1008-861x.2009.04.024

Qin, R., Li, X., Li, G., Tao, L., Li, Y., Sun, J., et al. (2011). Protection by tetrahydroxystilbene glucoside against neurotoxicity induced by MPP+: the involvement of PI3K/Akt pathway activation. Toxicol. Lett. 202, 1-7. doi: 10.1016/j.toxlet.2011.01.001

Reinoso, G., Allen, J. C. Jr., Au, W. L., Seah, S. H., Tay, K. Y., and Tan, L. C. (2015). Clinical evolution of Parkinson's disease and prognostic factors affecting motor progression: 9-year follow-up study. Eur. J. Neurol. 22, 457-463. doi: $10.1111 /$ ene.12476

Rocha, E. M., de Miranda, B., and Sanders, L. H. (2018). Alpha-synuclein: pathology, mitochondrial dysfunction and neuroinflammation in Parkinson's disease. Neurobiol. Dis. 109, 249-257. doi: 10.1016/j.nbd.2017.04.004
Rogers, G., Davies, D., Pink, J., and Cooper, P. (2017). Parkinson's disease: summary of updated NICE guidance. BMJ 358:j1951. doi: 10.1136/bmj.j1951

Sarkar, S., Raymick, J., and Imam, S. (2016). Neuroprotective and therapeutic strategies against Parkinson's disease: recent perspectives. Int. J. Mol. Sci. 17:E904. doi: 10.3390/ijms17060904

Sun, F. L., Zhang, L., Zhang, R. Y., and Li, L. (2011). Tetrahydroxystilbene glucoside protects human neuroblastoma SH-SY5Y cells against MPP+-induced cytotoxicity. Eur. J. Pharmacol. 660, 283-290. doi: 10.1016/j.ejphar.2011.03.046

Sun, R., Wang, K., Wu, D., Li, X., and Ou, Y. (2012). Protective effect of paeoniflorin against glutamate-induced neurotoxicity in PC12 cells via Bcl-2/Bax signal pathway. Folia Neuropathol. 50, 270-276. doi: $10.5114 /$ fn.2012.30527

Tian, Y.Y., An, L.J., Jiang, L., Duan, Y.L., Chen, J., and Jiang, B. (2006). Catalpol protects dopaminergic neurons from LPS-induced neurotoxicity in mesencephalic neuron-glia cultures. Life Sci. 80, 193-199. doi: 10.1016/j.lfs.2006.09.010

Wang, X.D. (1985). Standard for diagnosing and distinguishing Parkinson's disease and Parkinsonian syndromes. Chin. J. Neurol. 18:256. (in Chinese)

Wang, Y., Lin, X.M., and Zheng, G.Q. (2011). Traditional Chinese medicine for Parkinson's disease in China and beyond. J. Altern. Complement Med. 17, 385-388. doi: 10.1089/acm.2011.0025

Wang, Y., Xie, C. L., Lu, L., Fu, D. L., and Zheng, G. Q. (2012). Chinese herbal medicine paratherapy for Parkinson's disease: a meta-analysis of 19 randomized controlled trials. Evid. Based Complement. Alternat. Med. 2012:534861. doi: 10.1155/2012/534861

Wang, Y., Xie, C. L., Wang, W. W., Lu, L., Fu, D. L., Wang, X. T., et al. (2013). Epidemiology of complementary and alternative medicine use in patients with Parkinson's disease. J. Clin. Neurosci. 20, 1062-1067. doi: 10.1016/j.jocn.2012.10.022

Wang, Y. H., Xuan, Z. H., Tian, S., and Du, G. H. (2015). Echinacoside protects against 6-hydroxydopamine-induced mitochondrial dysfunction and inflammatory responses in PC12 cells via reducing ROS production. Evid. Based Complement Alternat. Med. 2015:189239. doi: 10.1155/2015/189239

Wen, L. L., He, Y., and Ji, L. (2015). Congwu Extinguish Wind Granule on movement of syndromes symptoms of Parkinson's disease and auality of life of intervention study. J. Liaoning Univ. Tradit. Chin. Med. 17, 130-132. doi: 10.13194/j.issn.1673-842x.2015.04.045

Yang, N. (2017). The Research on the Distribution of "Elements of Syndrome and Pathogenesis" of Parkinson's Disease and the Clinical Observation of Yishen Chuchan Decoction on Treating 40 cases. Dissertation, Nanjing: Nanjing University of Chinese Medicine (in Chinese).

Yang, W. T., Zheng, X. W., Chen, S., Shan, C. S., Xu, Q. Q., Zhu, J. Z., et al. (2017). Chinese herbal medicine for Alzheimer's disease: clinical evidence and possible mechanism of neurogenesis. Biochem. Pharmacol. 141, 143-155. doi: 10.1016/j.bcp.2017.07.002

Yu, T. (2016). Clinical Study on the Treatment of Parkinson's Disease With Syndrome of Wind Stirring due to Blood Stasis by Naokang Granules. Dissertation, Guangzhou: Guangzhou University of Chinese Medicine (in Chinese).

Zhang, G., Xiong, N., Zhang, Z., Liu, L., Huang, J., Yang, J., et al. (2015). Effectiveness of traditional Chinese medicine as an adjunct therapy for Parkinson's disease: a systematic review and meta-analysis. PLOS ONE 10: e0118498. doi: 10.1371/journal.pone.0118498

Zhang, J. X., Wang, R., Xi, J., Shen, L., Zhu, A.Y., Qi, Q., et al. (2017). Morroniside protects SK-N-SH human neuroblastoma cells against $\mathrm{H} 2 \mathrm{O} 2$-induced damage. Int. J. Mol. Med. 39, 603-612. doi: 10.3892/ijmm.2017.2882

Zhang, Q. S., Wang, Z. H., Zhang, J. L., Duan, Y. L., Li, G. F., and Zheng, D. L. (2016). Beta-asarone protects against MPTP-induced Parkinson's disease via regulating long non-coding RNA MALAT1 and inhibiting $\alpha$-synuclein protein expression. Biomed. Pharmacother. 83, 153-159. doi: 10.1016/j.biopha.2016. 06.017

Zhang, Y., Wang, Z. Z., Sun, H. M., Li, P., Li, Y. F., and Chen, N. H. (2014). Systematic review of traditional chinese medicine for depression in Parkinson's disease. Am. J. Chin. Med. 42, 1035-1051. doi: 10.1142/S0192415X14500657

Zhang, Z.X. (2006). The diagnosis of Parkinson's disease. Chin. J. Neurol. 39, 408-409. doi: 10.3760/j.issn:1006-7876.2006. 06.024 
Zhao, G. H., Meng, Q. G., Yu, X. D., Bai, J. Y., Luo, X. D., Yuan, C. X., et al. (2009). A multicentered randomized double-blinded controlled linical study on efficacy of Guiling Pa'an capsule in treating Parkinson's disease. Chin. J. Integer. Tradit. West Med. 29, 590-594. doi: 10.3321/j.issn:1003-5370.2009.07.006

Zhao, G. H., Sun, F., Feng, X. G., and Gao, M. (2013). Treatment of non-motor symptoms of Parkinson's disease patients of Gan-Shen deficiency syndrome by Guling Pa’an Granule:a multi-center double-blinded randomized controlled trial. Chin. J. Integer. Tradit. West Med. 33, 476-479 (in Chinese).

Zheng, G. Q. (2009). Therapeutic history of Parkinson's disease in Chinese medical treatises. J. Altern. Complement. Med. 15, 1223-1230. doi: $10.1089 / \mathrm{acm} .2009 .0101$
Conflict of Interest Statement: The authors declare that the research was conducted in the absence of any commercial or financial relationships that could be construed as a potential conflict of interest.

Copyright (๑) 2018 Shan, Zhang, Xu, Shi, Wang, Li, Lin and Zheng. This is an open-access article distributed under the terms of the Creative Commons Attribution License (CC BY). The use, distribution or reproduction in other forums is permitted, provided the original author(s) and the copyright owner(s) are credited and that the original publication in this journal is cited, in accordance with accepted academic practice. No use, distribution or reproduction is permitted which does not comply with these terms. 